\title{
Comparação entre overdenture e prótese total fixa sobre implante: revisão de literatura
}

Comparison between overdenture and implant-supported prosthesis: literature review Comparación entre overdenture y prótesis total fijada sobre implante: revisión de literatura

\author{
Larissa Augusta FERREIRA ${ }^{1}$ \\ Rafael dos Santos SILVA ${ }^{1}$ \\ Rodrigo Lorenzi POLUHA ${ }^{2}$
}

${ }^{I}$ Departamento de Odontologia, Universidade Estadual de Maringá-UEM. 87080-000 Maringá-PR, Brasil

${ }^{2}$ Departamento de Prótese Dentária, Faculdade de Odontologia de Bauru, Universidade de São Paulo-USP 17012-901 Bauru-SP, Brasil

\section{Resumo}

A overdenture e as próteses totais fixas sobre implante são importantes formas de reabilitação para pacientes edêntulos. A escolha para o melhor tratamento está de acordo com a indicação e o correto planejamento. O objetivo deste trabalho é comparar esses dois tipos de reabilitação em mandíbula edêntula. O comparativo entre overdenture e prótese total fixa sobre implantes quanto a satisfação geral do paciente sugere que ambas superam as expectativas de reabilitação, levando em conta o aspecto financeiro, estabilidade e higiene. Sendo este último fator mais vantajoso no quesito de facilidade para overdenture.

Descritores: Prótese Dentária; Implantes Dentários; Revestimento de Dentadura.

\section{Abstract}

Overdentures and fixed Implant-supported prosthesis are important forms of rehabilitation for edentulous patients. The choice for the best treatment is according to the indication and the correct planning. The objective of this study is to compare these two types of rehabilitation in the edentulous mandible. The comparison between overdenture and fixed Implant-supported prosthesis regarding general patient satisfaction suggests that both surpass expectations of rehabilitation, taking into account the financial aspect, stability, and hygiene. This last factor is more advantageous in the area of ease for overdenture.

Descriptors: Dental Prosthesis; Dental Implants; Denture, Overlay.

\section{Resumen}

Las overdentures y las prótesis totales fijas sobre el implante son importantes formas de rehabilitación para pacientes edéntulos. La elección para el mejor tratamiento está de acuerdo con la indicación y la correcta planificación. El objetivo de este trabajo es comparar estos dos tipos de rehabilitación en mandíbula edéntula. El comparativo entre sobredentadura y prótesis total fija sobre implantes en cuanto a la satisfacción general del paciente sugiere que ambas superan las expectativas de rehabilitación, teniendo en cuenta el aspecto financiero, estabilidad e higiene. Siendo este último factor más ventajoso en el aspecto de facilidad para overdenture.

Descriptores: Prótesis Dental; Implantes Dentales; Prótesis de Recubrimiento.

\section{INTRODUÇÃO}

As maiores queixas de pacientes edêntulos quanto as próteses totais convencionais estão relacionadas à estabilidade e retenção. Nesses casos, implantes osseointegrados são a solução para o sucesso da reabilitação bucal ${ }^{1}$. A prótese total fixa sobre implantes (protocolo) é a opção de escolha da maioria dos pacientes, porém nem sempre é possível sua confecção ${ }^{2}$. Apesar dessa preferência por próteses fixas, reabilitação removíveis como as overdentures são uma opção que apresenta eficácia e previsibilidade além de serem menos onerosas com relação as próteses fixas ${ }^{1,3}$. O plano de tratamento reabilitador deve ser individualizado para cada paciente relacionando suas necessidades físicas e psicológicas com as condições ósseas, relação maxilo-mandibular, estética, capacidade de higienização, fatores congênitos ou adquiridos e tolerância às próteses totais ${ }^{4}$. $\mathrm{O}$ presente trabalho tem como objetivo revisar a literatura e comparar os dois tipos de reabilitação protética em mandíbula edêntula: overdentures e prótese total fixa sobre implantes.

\section{MATERIAL E MÉTODO}

Foi realizada busca eletrônica nas bases de dados Lilacs, Pubmed/Medline, Scielo, Portal Ebsco, Biblioteca Cochrane e ScienceDirect, cruzando-se os seguintes descritores: "overdenture", "dental implants", "mandible prosthesis", e "fixed prosthesis". Foram incluídos somente artigos de revisão de literatura, revisão sistemática, metanálise, e estudos clínicos randomizados publicados no período de janeiro de 1993 a novembro de 2018.

\section{RESULTADOS E DISCUSSÃO}

- Indicações para cada Reabilitação

A seguir, segue uma descrição em tópicos das indicações de cada tipo de reabilitação abordada no estudo ${ }^{5}$.

- Overdenture

- Indicada para pacientes que apresentam maior dificuldade motora (devido a fácil higienização);

- Pacientes com grande perda de suporte labial;

- Baixa condição socioeconômica;

- 4 ou menos implantes instalados.

- Prótese total fixa sobre implantes

- Pacientes com pouca perda de tecidos de suporte;

- Suporta altas cargas mastigatórias;

- Quantidade óssea necessária para instalação do número necessário de implantes (4 ou mais);

- Situações que requerem alta estabilidade protética

- Overdentures - prótese total fixa sobre implantes

Diversos fatores foram comparados entre overdentures e prótese total fixa sobre implantes. A qualidade e quantidade óssea foram apontadas em alguns trabalhos como os grandes influenciadores na retenção e estabilidade da reabilitação escolhida ${ }^{6,7}$. A indicação de cada procedimento deve ser individualizada e baseada nos aspectos clínicos e psicológicos do paciente, dando importância para sua 
situação econômica ${ }^{8}$. O planejamento para uma reabilitação com overdenture ou protocolo deve se basear na carga mecânica em que estas estarão sujeitas ${ }^{9}$. Também devem ser levados em consideração a quantidade e qualidade óssea remanescente na arcada, tanto em altura quanto largura, para assim, ter os corretos posicionamento e quantidade dos implantes, o espaço inter arcos, assim como o suporte labial, linha do sorriso, condições da mucosa oral, relação esquelética maxilo-mandibular, e contorno do rebordo alveolar ${ }^{4,7}$.

Overdentures implanto retidas podem ser instaladas em casos de quatro ou menos implantes na arcada dentária. Próteses tipo protocolo são indicadas nos casos onde é possível instalar a quantidade suficiente de implantes na distância $\operatorname{correta}^{10,}{ }^{11}$. Muitos benefícios foram encontrados através de uma reabilitação com overdenture, como uma boa retenção e estabilidade, assim como um custo reduzido, fácil manuseio e baixa invasividade ${ }^{5,12}$. Outros estudos relataram vantagens quanto a overdenture relacionadas à reposição do suporte labial, contribuindo para a restauração do perfil facial, e a facilidade para higienização, benéfica para pacientes com dificuldades motoras ${ }^{4,7,13}$.

A prótese total fixa sobre implantes é considerada pela maioria dos clínicos como tratamento padrão para reabilitar mandíbula edêntula por sua alta estabilidade, já que a prótese é fixa nos implantes ${ }^{5,14}$. As próteses fixas são indicadas em casos de pouca perda dos tecidos de suporte ${ }^{5}$. Outro fator favorável em relação às próteses fixas é que estas apresentam uma infraestrutura mais resistente às cargas mastigatórias ${ }^{4}$. No que diz respeito à estética e fatores funcionais, tanto as reabilitações com overdenture quanto com prótese fixa tipo protocolo atingiram alta satisfação entre os pacientes, não apresentando diferença estatística significante entre os grupos avaliados ${ }^{15^{3}}$.

Tanto com overdenture quanto com as próteses fixas, os pacientes relataram uma boa reestruturação fonética. Entretanto, nos casos de reabilitação com protocolo em que haviam perda óssea, foram observados escape de ar e saliva, o que não aconteceu durante $\mathrm{o}$ uso das overdentures ${ }^{7}$. Segundo Hemmings ${ }^{16}$ foram observadas muitas complicações durante o primeiro ano de uso das próteses fixas em relação à overdenture, destacando principalmente inflamação periimplantar ou hiperplasia gengival. Isto deve se dar ao fato da overdenture ser mais fácil de higienizar, especialmente se ocorreu um mau posicionamento dos implantes durante a fase cirúrgica, em detrimento das próteses tipo protocolo. Souza et al. ${ }^{15}$ em 2016, avaliaram o nível de satisfação dos pacientes que possuíam prótese fixa tipo protocolo e overdenture. De acordo com a satisfação quanto a estabilidade da prótese e quanto ao tratamento em geral, não houve diferença estatística relevante entre os dois tipos de reabilitação. O mesmo ocorreu quanto à fonética, função mastigatória, dor após o procedimento e melhora da autoestima do paciente.

CONCLUSÃO

O comparativo entre overdenture e prótese total fixa sobre implantes quanto a satisfação geral do paciente sugere que ambas superam as expectativas de reabilitação, levando em conta o aspecto financeiro, estabilidade e higiene. Sendo este último fator mais vantajoso no quesito de facilidade para overdenture.

\section{REFERÊNCIAS}

1. Chao YL, Meijer HJ, Van Oort RP, Versteegh PA. The incomprehensible success of the implant stabilised overdenture in the edentulous mandible: a literature review on transfer of chewing forces to bone surrounding implants. Eur J Prosthodont Restor Dent. 1995;3(6):255-61.

2. DeBoer J. Edentulous implants: overdenture versus fixed. J Prosthet Dent. 1993;69(4):386-90.

3. Saavedra G, Barbosa SH, Kimpara ET. Influência do angulo de inserção na degradação da retenção do o'ring em overdentures. Implant News. 2007;4(3):249-53.

4. Gallina C, Viegas VN. Overdentures e próteses fixas para reabilitação com implantes em maxila edêntula. Rev Odontol Univ Cid São Paulo 2007;19(1):61-7.

5. Telles D. Próteses fixas sobre implantes. Quintessence: São Paulo; 2014.

6. Assunção WG, Zardo GG, Delben JA, Barão VA. Comparing the efficacy of mandibular implantretained overdentures and conventional dentures among elderly edentulous patients: satisfaction and quality of life. Gerodontology. 2007; 24(4):235-38.

7. Almeida HCR, Santana ETS, Santos NAT, Moraes PKM, Araújo YRF, Gerbi MEM. Clinical aspects in the treatment planning for rehabilitation with overdenture and protocol-type prosthesis. RGO Rev Gaúch Odontol. 2015;63(3):271-76.

8. Fitzpatrick B. Standard of care for the edentulous mandible: a systematic review. J Prosthet Dent. 2006;95(1):71-8.

9. Agliardi E, Panigatti S, Clericò M, Villa C, Malò P. Immediate rehabilitation of the edentulous jaws with full fixed prostheses supported by four implants: interim results of a single cohort prospective study. Clin Oral Implants Res. 2010;21(5):459-65.

10.Heydecke G, Boudrias P, Awad MA, De Albuquerque RF, Lund JP, Feine JS. Withinsubject comparisons of maxillary fixed and removable implant prostheses: Patient satisfaction and choice of prosthesis. Clin Oral Implants Res. 2003;14(1):125-30. 


\section{CONFLITO DE INTERESSES}

Os autores declaram não haver conflitos de interesse.

AM, Preoteasa CT. Two implant overdenture - the first alternative treatment for patients with complete edentulous mandible. J Med Life. 2011;4(2):207-9.

12.Poluha RL, De Moraes Melo Neto CL, Milhomens de Sousa B, Moura FL, Sábio S. Overdenture na reabilitação de paciente desdentado. Herediana. 2016;26(3):151-161.

13.Borges Tde F, Mendes FA, de Oliveira TR, do Prado CJ, das Neves FD. Overdenture with immediate load: mastication and nutrition. $\mathrm{Br} \mathrm{J}$ Nutr. 2011;105(7):990-94.

14.Zarb GA, Schimitt A. The edentulous predicament. I: A prospective study os the effectiveness of implant-supported fixed prostheses. J Am Dent Assoc. 1996;127(1):59-65.

15.de Souza FI, de Souza Costa A, Dos Santos Pereira R, Dos Santos PH, de Brito RB Jr, Rocha EP. Assessment of Satisfaction Level of Edentulous Patients Rehabilitated with ImplantSupported Prostheses. Int J Oral Maxillofac Implants. 2016;31(4):884-90.

16.Hemmings KW, Schmitt A, Zarb GA. Complications and maintenance requirements for fixed prostheses and overdentures in the edentulous mandible: a 5-year report. Int J Oral Maxillofac Implants. 1994;9(2):191-96.
Submetido em 02/04/2019

Aceito em 27/08/2019

\section{AUTOR PARA CORRESPONDENCIA}

\section{Rodrigo Lorenzi Poluha}

rodrigopoluha@gmail.com 